Geometry $\&$ Topology

Volume 9 (2005) 163-178

Published: 6 January 2005

Corrected: 7 March 2005

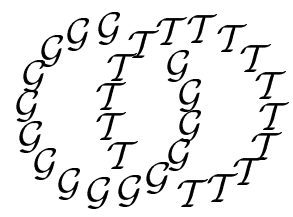

\title{
Warped cones and property A
}

\author{
John Roe \\ Department of Mathematics, Penn State University \\ University Park PA 16802, USA \\ Email: roe@math.psu.edu
}

\begin{abstract}
We describe a construction (the 'warped cone construction') which produces examples of coarse spaces with large groups of translations. We show that by this construction we can obtain many examples of coarse spaces which do not have property A or which are not uniformly embeddable into Hilbert space.
\end{abstract}

AMS Classification numbers Primary: 53C20

Secondary: $43 \mathrm{~A} 07,53 \mathrm{C} 12,20 \mathrm{~F} 69$

Keywords: Coarse geometry, amenable action, property A, warped cone

Proposed: Martin Bridson

Seconded: Steven Ferry, Benson Farb
Received: 17 March 2004

Accepted: 27 December 2004

(C) Geometry $\mathcal{E}^{\mathcal{G}}$ Topology Publications 
The purpose of this paper is to explore some aspects of the geometry of those coarse spaces which may be created from compact foliated manifolds, or from discrete group actions on compact metric spaces, by way of the warped cone construction. This construction was introduced in [6] (see also [7]) and some of its properties were predicted there. Since those papers were written, workers in coarse geometry such as $\mathrm{Yu}$ [10] have drawn attention to the importance of coarse (or uniform) embeddability in Hilbert space, and the related 'coarse amenability' property $A$. We shall show that the warped cone construction is sufficiently flexible to create natural new examples both of spaces which have, and of spaces which do not have, property A. In a sequel to this paper 2] we will show that the class of warped cones also includes counterexamples to the coarse Baum-Connes conjecture.

The first section of this paper reviews in detail the construction of warped cones (the article [6] contains no proofs). In the second section we briefly review and generalize the definition of property $\mathrm{A}$; for the purposes of this paper it is necessary to extend the definition from the context of uniformly discrete spaces, in which it is presented in the literature, to that of more general coarse spaces. In the third section we show that the warped cone over an amenable action is a space with property A. The fourth and final section contains a partial converse: if the warped cone over a particular kind of action has property $\mathrm{A}$, then the action itself is amenable. This allows us to construct warped cones that do not have property $\mathrm{A}$, or that are not uniformly embeddable into Hilbert space. There is a striking parallel between the theory of warped cones and the theory of the 'box spaces' of [8, Chapter 11]; this will be brought out in the main text.

It is a pleasure to thank Eric Guentner and Nigel Higson for illuminating discussions.

\section{Definition and basic properties of warped cones}

Let $(X, d)$ be a proper metric space. We are going to consider $X$ as a coarse space (see [3] for the definition), so that only the 'large scale' properties of the metric on $X$ will be relevant to us. Recall that a metric space is proper if closed bounded subsets are compact.

1.1 Example We will be particularly concerned throughout this paper with the case $X=\mathcal{O} Y$, the open cone on a compact space $Y$. We will assume that $Y$ is a 'nice' space such as a smooth manifold or a finite simplicial complex. 
The open cone $X=\mathcal{O} Y$ may be defined in two equivalent ways, which we state for $Y$ a manifold:

(a) Embed $Y$ smoothly in a high-dimensional sphere $S^{N-1}$, and let $X$ be the union of all the rays through the origin in $\mathbb{R}^{N}$ that meet the embedded copy of $Y$; equip $X$ with the metric (distance function) induced from $\mathbb{R}^{N}$.

(b) Let $X=Y \times[1, \infty)$ as a manifold 1 , equipped with the Riemannian metric $t^{2} g_{Y}+g_{\mathbb{R}}$, where $g_{Y}$ is a Riemannian metric on $Y, g_{\mathbb{R}}$ is the standard Riemannian metric on $\mathbb{R}$, and $t$ is the coordinate on $\mathbb{R}$.

(There are obvious 'piecewise linear' versions of both constructions in case $Y$ is a finite complex.) It is a straightforward exercise to show that up to coarse equivalence these constructions are independent of the choices involved and, moreover, that both constructions yield coarsely equivalent spaces $X$.

1.2 Definition A map $f: X \rightarrow X$ is a translation if it is a bijection and if the supremum $\sup _{x \in X} d(x, f(x))$ is finite.

The translations of a coarse space form a group which is closely related to the coarse structure of the space.

1.3 Example Let $\Gamma$ be a discrete group equipped with a right invariant metric. Then the left action of $\Gamma$ on itself is an action by translations.

Suppose now that $X$ is a coarse space and that $\Gamma$ is a group acting 2 on $X$ (the example of particular interest to us is $X=\mathcal{O} Y, \Gamma$ a group of diffeomorphisms of $Y$.) It will typically not be the case that $\Gamma$ acts by translations on $X$ and we want to modify the metric on $X$ in such a way as to ensure that the action of $\Gamma$ becomes an action by translations. We will describe this process as warping $X$ by $\Gamma$.

1.4 Lemma Let $\mathcal{F}$ be a family of metrics on a set $X$. If the supremum

$$
d\left(x, x^{\prime}\right)=\sup \left\{\delta\left(x, x^{\prime}\right): \delta \in \mathcal{F}\right\}
$$

is finite for each pair $\left(x, x^{\prime}\right) \in X \times X$, then it defines a metric on $X$.

The proof is elementary.

\footnotetext{
${ }^{1}$ Here we have - for simplicity - omitted a neighborhood of the cone point. Our interest is in the other end of the cone, in the large scale behavior of the geometry, so that the treatment of the singularity does not matter.

${ }^{2}$ Actions of a group on a space will be taken on the left.
} 
1.5 Definition Let $(X, d)$ be a proper metric space and let $\Gamma$ be a group acting by homeomorphisms on $X$, provided with a finite generating set $S$. The warped metric $d_{\Gamma}$ on $X$ is the greatest metric that satisfies the inequalities

$$
d_{\Gamma}\left(x, x^{\prime}\right) \leq d\left(x, x^{\prime}\right), \quad d_{\Gamma}(x, s x) \leq 1 \forall s \in S .
$$

The existence and uniqueness of the warped metric follow easily from the Lemma above - the warped metric is the supremum of the family $\mathcal{F}$ of metrics satisfying the inequalities that appear in its definition. More constructively, we can say:

1.6 Proposition Let $x, y \in X$. For $\gamma \in \Gamma$, let $|\gamma|$ denote the word length of $\gamma$ relative to the generating set $S$. The warped distance from $x$ to $y$ is the infimum of all sums

$$
\sum d\left(\gamma_{i} x_{i}, x_{i+1}\right)+\left|\gamma_{i}\right|
$$

taken over all finite sequences $x=x_{0}, x_{1}, \ldots, x_{N}=y$ in $X$ and $\gamma_{0}, \ldots, \gamma_{N-1}$ in $\Gamma$. Moreover, if $d(x, y) \leq k$, then one can find a finite sequence as above with $N=k+1$ such that the infimum is attained.

Proof The expression in the proposition defines a metric, call it $\delta$, that belongs to the family of metrics $\mathcal{F}$ mentioned above, and thus $\delta \leq d_{\Gamma}$ by definition. On the other hand, $d_{\Gamma} \leq \delta$ by the triangle inequality. Thus the two metrics are equal. This proves the first part of the proposition.

For the second part, note that in order for the sum above to be $\leq k+1$, no more than $k+1$ group elements $\gamma_{i}$ can differ from the identity. If say $\gamma_{i}$ is equal to the identity, then $\gamma_{i}$ and $x_{i}$ may be omitted from the chain and by the triangle inequality the sum will only decrease. Thus every chain may be shortened to one having $N \leq k+1$. The set of such chains beginning at $x$ and ending at $y$ is compact (by the properness of the metric space $X$ ), so the infimum is attained.

1.7 Proposition The warped metric is a proper metric. The coarse structure induced by the warped metric does not depend on the choice of generating set $S$ for $\Gamma$, nor on the choice of metric $d$ within the coarse structure of $X$.

Proof For a subset $K$ of $X$, define $N_{k}(K)$ to be

$$
N(K)=\{x \in X: d(x, \gamma y) \leq 1 \text { for some } y \in K,|\gamma| \leq 1\} ;
$$


that is, $N_{k}(K)$ is the union of the closed $k$-neighborhoods of translates of $K$ by generators of the group, together with the closed $k$-neighborhood of $K$ itself. Since $X$ is proper and $\Gamma$ acts by homeomorphisms, the operation $N_{k}$ preserves compactness: if $K$ is compact, then so is $N_{k}(K)$. It follows from Proposition [1.6] that the warped $k$-ball around a point $p$ is contained in the compact set $\left(N_{k}\right)^{k+1}\{p\}$. This proves properness.

Consider the abstract coarse structure $\mathcal{S}$ on $X$ generated by the controlled sets for the original metric on $X$ together with the sets $\{(x, \gamma x): \gamma \in \Gamma\}$. This structure is countably generated and hence metrizable [8, Theorem 2.55]. The generators for $\mathcal{S}$ are all controlled for the warped metric, so every $\mathcal{S}$-controlled set is controlled for the warped metric. On the other hand, by Proposition 1.6 every set controlled for the warped metric is $\mathcal{S}$-controlled. Thus the warped metric describes the coarse structure $\mathcal{S}$. Since the definition of $\mathcal{S}$ does not involve the choice of metric on $X$ nor the choice of generating set for $\Gamma$, any two warped metrics are coarsely equivalent.

We can now make the key definition of this paper.

1.8 Definition Let $Y$ be a smooth compact manifold, or a finite simplicial complex, and let $\Gamma$ be a finitely generated group acting by homeomorphisms on $Y$. The warped cone $\mathcal{O}_{\Gamma} Y$ is the coarse space obtained by warping $\mathcal{O} Y$ along the induced $\Gamma$-action.

Typically the metric structure of $\mathcal{O}_{\Gamma} Y$ is difficult to envisage. Each crosssection $Y_{t}$ is a copy of $Y$ with the metric modified in the following manner: first the ambient metric of $Y$ is multiplied by $t$, and then 'shortcuts' or 'wormholes' are introduced along the generators of the group action, each of which 'costs' one unit of distance to travel through. It is clear that as $t$ increases, the distortion introduced by the warping becomes progressively more severe. Nevertheless, the $Y_{t}$ do increase in size:

1.9 Lemma With notation as above, the diameter of $Y_{t}$ tends to infinity as $t \rightarrow \infty$, unless $Y$ is a finite set and $\Gamma$ acts transitively on it. Moreover, if $\Gamma$ acts by diffeomorphisms on the compact manifold $Y$, the diameter of $Y_{t}$ grows at least as fast as $\log t$.

Proof The proof of the first part is left to the reader. For the second part, use elementary estimates of volume: the volume, in some fixed Riemannian metric 
on $Y$, of the warped $d$-ball in $Y_{t}$ about some point, is of order at most the number of group elements of word length $\leq d$ times the maximum volume of a set of the form $\gamma(B)$, where $B$ is a $d / t$-ball in $Y$ and $\gamma$ is a word in $\Gamma$ of length at most $d$. This volume is at most $C e^{k d}(d / t)^{n}$, where $C$ and $k$ are constants and $n$ is the dimension of $Y$. If this quantity is less than the volume of $Y$, then $Y_{t}$ has diameter $\geq d$; the logarithmic growth follows.

1.10 Proposition Suppose that $\Gamma$ acts on $Y$ through Lipschitz homeomorphisms. Then the space $X=\mathcal{O}_{\Gamma} Y$ has bounded geometry.

Proof (I am grateful for Graham Niblo and Nick Wright for pointing out an error in the proof given in the first version of this paper.) Let $X$ be a metric space and let $\varepsilon>0$. By definition, the $\varepsilon$-capacity of a subset $A$ of $X$ is the maximum cardinality of an $\varepsilon$-separated subset of $A$. We shall say that $X$ has bounded geometry if there is an $\varepsilon>0$ such that for every $r>0$ there is a $s>0$ such that each $r$-ball in $X$ has $\varepsilon$-capacity at most $s$. An open cone $X=\mathcal{O} Y$ on a manifold or finite complex has bounded geometry, as one sees most easily by regarding it as a metric subspace of a Euclidean space.

Suppose that $X$ has bounded geometry. We will say for the purposes of this proof that a subset of $X$ has size $\leq(p, q)$ if it is the union of at most $p$ subsets of $X$ each of which has diameter at most $q$. Clearly, for each $(p, q)$ there is $C=C(p, q)$ such that any subset of size $\leq(p, q)$ has $\varepsilon$-capacity at most $C$.

Now let $X=\mathcal{O} Y$, where $\Gamma$ acts on $Y$ (and therefore on $X$ ) through Lipschitz homeomorphisms. From its definition, there exist constants $C$ and $c$ such that if $S$ has size $\leq(p, q)$ (relative to the unwarped metric on $X)$, then $N_{k}(S)$ has size $\leq(C p, c q+2 k) ; C$ is the number of generators for the group, and $c$ is the maximum Lipschitz constant for the action of a generator. But it was shown in the proof of Proposition 1.7 that the $d_{\Gamma}$-ball of radius $k$ around any point $p$ is contained in $\left(N_{k}\right)^{k+1}\{p\}$. It therefore follows by induction that the $\varepsilon$-capacity (in the $d$-metric) of any such ball is bounded. However, $d_{\Gamma} \leq d$ and therefore the capacity of this ball in the $d_{\Gamma}$-metric is at most equal to its capacity in the $d$-metric. Bounded geometry now follows.

1.11 Remark In [6] we defined warped cones not for group actions but for foliations. We review this construction briefly. Let $V$ be a compact smooth manifold equipped with a foliation $F$. Choose a 'normal bundle' $N$ to the foliation, that is a complementary subbundle to $T F$ in $T M$. Choose Euclidean 
metrics $g_{N}$ in $N$ and $g_{F}$ in $T F$. The foliated warped cone $\mathcal{O}_{F} V$ is the manifold $V \times[0, \infty) / V \times\{0\}$ equipped with the distance function induced for $t \geq 1$ by the Riemannian metric $g_{\mathbb{R}}+g_{F}+t^{2} g_{N}$. As in our earlier discussion, the structure near $t=0$ does not much matter from the point of view of coarse geometry; we imagine that the space $\mathcal{O}_{F} V$ has been equipped with some arbitrary path metric in this region. One sees without difficulty that the coarse structure of $\mathcal{O}_{F} V$ does not depend on the choice of normal bundle $N$, nor does it depend on the choice of metrics $g_{F}$ and $g_{N}$ (compare Proposition 1.7).

Foliations are often constructed by 'suspending' group actions. For foliations constructed in this way, there is a relationship between our two notions of warped cone. Let $\Gamma$ be a group acting freely by diffeomorphisms on a manifold $M$, and suppose that $\Gamma$ has a classifying space $B \Gamma$ which is itself a compact manifold. Form the balanced product $V=M \times_{\Gamma} E \Gamma$. This is a compact manifold. The foliation of $M \times E \Gamma$ by copies of $E \Gamma$ descends to a foliation $F$ of $V$ whose leaves are also copies of $E \Gamma$ (since we are assuming that the action is free). The manifold $M$ may be embedded in $V$ as a transversal to the foliation.

1.12 Lemma In the above situation, the embedding of $M$ as a transversal in $V$ induces a coarse equivalence $\mathcal{O}_{\Gamma}(M) \rightarrow \mathcal{O}_{F}(V)$.

We leave the proof to the reader.

\section{Property A for general spaces}

For a locally compact, $\sigma$-compact topological space $X$ (e.g. a bounded geometry metric space), let $\operatorname{Prob}(X)$ denote the space of Radon probability measures on $X$. It is the state space of the $C^{*}$-algebra $C_{0}(X)$ and as such it carries two topologies: the norm topology and the weak- $*$ topology. We will use both in the definition below.

2.1 Definition Let $X$ be a bounded geometry proper metric space. We say that $X$ has property $A$ if there exists a sequence of weak-* continuous maps $f_{n}: X \rightarrow \operatorname{Prob}(X)$ such that

(i) for each $n$ there is an $r$ such that, for each $x$, the measure $f_{n}(x)$ is supported within $B(x ; r)$, and 
(ii) for each $s>0$, as $n \rightarrow \infty$,

$$
\sup _{d(x, y)<s}\left\|f_{n}(x)-f_{n}(y)\right\| \rightarrow 0 .
$$

For brevity we will refer to (i) above as the property of being uniformly localized.

If $X$ is a discrete metric space of bounded geometry, then this is Yu's definition as reformulated in [4, Lemma 3.5]. Moreover, there is a simple reduction to the discrete case. A uniformly discrete subset $Z \subseteq X$ will be called a lattice in $X$ if there is $R>0$ such that $X=\bigcup_{z \in Z} B(z ; R)$. The inclusion $Z \rightarrow X$ is then a coarse equivalence.

2.2 Lemma $A$ (bounded geometry proper metric) space $X$ has property $A$ if and only if some (and hence every) lattice in $X$ has property $A$.

Proof Let $Z$ be a lattice in $X$ and let $\left\{\varphi_{z}\right\}$ be a partition of unity on $X$ subordinate to a cover of $X$ by balls $B(z ; R), z \in Z$.

Suppose that $X$ has property $A$ and let maps $f_{n}: X \rightarrow \operatorname{Prob}(X)$ be as in the definition 2.1. Define maps $g_{n}: Z \rightarrow \operatorname{Prob}(Z)$ by

$$
g_{n}(z)=\sum_{w \in Z}\left(\int \varphi_{w} d f_{n}(z)\right) \delta_{w},
$$

where $\delta_{w}$ denotes the Dirac mass at $w$. This is a probability measure, uniformly localized near $z$, and we have

$$
\begin{aligned}
&\left\|g_{n}(z)-g_{n}\left(z^{\prime}\right)\right\|=\sum \mid \int \varphi_{w} d\left[f_{n}(z)-f_{n}\left(z^{\prime}\right)\right] \mid \\
& \leq \sum \int \varphi_{w} d\left|f_{n}(z)-f_{n}\left(z^{\prime}\right)\right|=\left\|f_{n}(z)-f_{n}\left(z^{\prime}\right)\right\| .
\end{aligned}
$$

This shows that $Z$ has property A.

Conversely, suppose that $Z$ has property A and let $g_{n}: Z \rightarrow \operatorname{Prob}(Z)$ be a defining sequence of maps. We may consider probability measures on $\mathrm{Z}$ as probability measures on X (linear combinations of Dirac measures). Set

$$
f_{n}(x)=\sum_{z} \varphi_{z}(x) g_{n}(z)
$$

where $g_{z}$ is considered as a probability measure on $X$. Then $f_{n}(x)$ is a probability measure, uniformly localized near $x$, depending continuously on $x \in X$, and we have

$$
\left\|f_{n}(x)-f_{n}\left(x^{\prime}\right)\right\| \leq \sup _{z, z^{\prime} \in B(x ; R) \cup B\left(x^{\prime} ; R\right)}\left\|g_{n}(z)-g_{n}\left(z^{\prime}\right)\right\|
$$


since both $f_{n}(x)$ and $f_{n}\left(x^{\prime}\right)$ are convex combinations of the measures $g_{n}(z)$, for $z \in B(x ; R) \cup B\left(x^{\prime} ; R\right)$. This shows that $X$ has property A.

It is known in the discrete case that there is an alternative characterization of property $\mathrm{A}$ in terms of kernels of positive type. We will also need the continuous analogue of this characterization. Recall that a continuous function $k: X \times X \rightarrow \mathbb{R}$ is a (continuous) kernel of positive type if

$$
\sum_{i, j=1^{n}} \lambda_{i} \lambda_{j} k\left(x_{i}, x_{j}\right) \geq 0
$$

for all $n=1,2, \ldots$ and all $n$-tuples $\lambda_{1}, \ldots, \lambda_{n}$ in $\mathbb{R}$ and $x_{1}, \ldots, x_{n}$ in $X$.

A kernel $k$ has controlled support if there is $R>0$ such that $k\left(x, x^{\prime}\right)=0$ whenever $d\left(x, x^{\prime}\right)>R$.

2.3 Proposition The (bounded geometry proper metric) space $X$ has property $A$ if and only if there is a sequence $\left\{k_{n}\right\}$ of continuous positive definite kernels such that

(a) $\left|k_{n}\left(x, x^{\prime}\right)\right| \leq 1$ for all $x, x^{\prime}$;

(b) each $k_{n}$ has controlled support;

(c) $k_{n}\left(x, x^{\prime}\right) \rightarrow 1$ as $n \rightarrow \infty$, uniformly on each controlled set $\left\{\left(x, x^{\prime}\right)\right.$ : $\left.d\left(x, x^{\prime}\right)<r\right\}$.

Proof We shall take this proposition for granted in the uniformly discrete case (see [8] or 9] for the details) so our task is simply to show that $X$ has the three-part property (temporarily call it ' $\mathrm{P}$ ') of the Proposition if and only if some lattice $Z$ in $X$ has it.

If $X$ has $\mathrm{P}$, then we may simply restrict the positive definite kernels $k_{n}$ to $Z$ to show that $Z$ has $\mathrm{P}$ also.

Conversely, suppose that $Z$ has P. Let $\left\{\varphi_{z}\right\}$ be a partition of unity subordinate to some cover $B(z ; R)$, as in the proof of the previous proposition. If $\ell_{n}$ is a sequence of positive definite kernels exhibiting property $\mathrm{P}$ for $Z$, then one checks without difficulty that

$$
k_{n}\left(x, x^{\prime}\right)=\sum_{z, z^{\prime}} \ell_{n}\left(z, z^{\prime}\right) \varphi_{z}(x) \varphi_{z^{\prime}}\left(x^{\prime}\right)
$$

is a sequence of such kernels exhibiting property $\mathrm{P}$ for $X$.

It will be helpful to consider an apparent weakening of property $A$. 
2.4 Definition Let $X$ be a bounded geometry proper metric space. We say that $X$ has property $A$ at infinity if there exists a sequence of weak- $*$ continuous maps $f_{n}: X \rightarrow \operatorname{Prob}(X)$ such that

(i) for each $n$ there is an $r$ such that, for each $x$, the measure $f_{n}(x)$ is supported within $B(x ; r)$, and

(ii) for each $s>0$, as $n \rightarrow \infty$,

$$
\inf _{K \subseteq X \text { compact }} \sup _{d(x, y)<s, x, y \notin K}\left\|f_{n}(x)-f_{n}(y)\right\| \rightarrow 0 .
$$

Clearly, if a space has property A, it has property A at infinity. It is useful to observe that the converse is true also.

2.5 Lemma If a space $X$ has property $A$ at infinity, then it has property $A$.

Proof Let $\left\{f_{n}\right\}$ be a sequence of maps provided by the definition of property A at infinity. We are going to construct a sequence $g_{m}$ of weak-* continuous maps $X \rightarrow \operatorname{Prob}(X)$ which are uniformly localized and satisfy

$$
\sup _{d(x, y) \leq m}\left\|g_{m}(x)-g_{m}(y)\right\| \leq m^{-1} .
$$

To do so, fix $m$ and then choose $n$ so large that for some compact $K \subseteq X$,

$$
\sup _{d(x, y)<m, x, y \notin K}\left\|f_{n}(x)-f_{n}(y)\right\| \leq(4 m)^{-1} ;
$$

we can do this by definition of property $A$ at infinity. Fix a function $\varphi: X \rightarrow$ $[0,1]$ such that $\varphi(x)=1$ if $x \in K, \varphi(x)=0$ if $d(x, K)>5 m^{2}$, and $\varphi$ is Lipschitz with Lipschitz constant less than $(2 m)^{-2}$; for instance, $\varphi$ might be a suitable function of $d(x, K)$. Let $\delta_{K}$ be the Dirac measure supported at some (arbitrarily chosen) point of $K$. Now put

$$
g_{m}(x)=\varphi(x) \delta_{K}+(1-\varphi(x)) f_{n}(x) .
$$

It is clear that $g_{m}$ is a uniformly localized map from $X$ to $\operatorname{Prob}(X)$. Moreover if $d(x, y)<m$ then

$$
\left\|g_{m}(x)-g_{m}(y)\right\| \leq \frac{1}{4 m}+\frac{1}{4 m}+\left\|f_{n}(x)-f_{n}(y)\right\| \leq \frac{3}{4 m}
$$

and so we are done. 


\section{Warped cones over amenable actions}

3.1 Proposition Let $(X, d)$ be a bounded geometry coarse space that has property $A$, and let $\Gamma$ be an amenable group acting on $X$ (by coarse maps). Then the warped space $\left(X, d_{\Gamma}\right)$ has property $A$ also.

3.2 Corollary Let $Y$ be a compact manifold (or finite simplicial complex) and let $\Gamma$ be an amenable group acting by Lipschitz homeomorphisms on $Y$. Then the warped cone $\mathcal{O}_{\Gamma}(Y)$ has property $A$.

Proof Let $f_{n}: X \rightarrow \operatorname{Prob}(X)$ be the functions that are provided by the definition of property A. Let $\mu_{n}$ be a Følner sequence in $\operatorname{Prob}(\Gamma)$, by which we mean that $\left\{\mu_{n}\right\}$ is a sequence of finitely supported probability measures on $\Gamma$ such that, for each fixed $\gamma \in \Gamma$, the difference $\mu_{n}-\gamma \cdot \mu_{n}$ tends to zero in norm as $n \rightarrow \infty, 3$

For fixed $m>0$ choose $n_{1}$ so large that $\left\|\mu_{n_{1}}-\gamma \cdot \mu_{n_{1}}\right\|<(2 m)^{-2}$ for all $\gamma \in \Gamma$ having $|\gamma| \leq m$. Let $R$ be defined by

$$
R=\sup \left\{d(\gamma x, \gamma y): d(x, y) \leq m, \gamma \in \operatorname{Supp}\left(\mu_{n_{1}}\right)\right\}
$$

$R$ is finite because the supremum is taken over a finite set of coarse maps. Finally choose $n_{2}$ so large that $\left\|f_{n_{2}}(x)-f_{n_{2}}(y)\right\|<(2 m)^{-2}$ whenever $d(x, y) \leq$ $R$. Define a weak-* continuous function $g_{m}$ from $X$ to $\operatorname{Prob}(X)$ by convolving $\mu_{n_{1}}$ with $f_{n_{2}}$ :

$$
g_{m}(x)=\sum_{\gamma \in \Gamma} \mu_{n_{1}}\{\gamma\} f_{n_{2}}(\gamma x)
$$

It is clear from the construction of the warped metric $d_{\Gamma}$ that $g_{m}$ is $d_{\Gamma^{-}}$ uniformly localized; in fact $g_{m}(x)$ is supported within the $d_{\Gamma}$-ball around $x$ of radius

$$
\inf \left\{r: \operatorname{Supp}\left(f_{n_{2}}(x)\right) \subseteq B_{d}(x ; r) \forall x\right\}+\max \left\{|\gamma|: \gamma \in \operatorname{Supp}\left(\mu_{n_{1}}\right)\right\},
$$

the first term being finite by the definition of property $\mathrm{A}$ and the second being finite by our assumption that $\mu_{n_{1}}$ is finitely supported. We will show that $\left\|g_{m}(x)-g_{m}(y)\right\| \leq 1 / m$ for $d_{\Gamma}(x, y)<m$; this will complete the proof that the maps $\left\{g_{m}\right\}$ are a sequence realizing property $\mathrm{A}$ for the $d_{\Gamma}$-metric.

\footnotetext{
${ }^{3}$ The action of $\Gamma$ on $\operatorname{Prob}(\Gamma)$ is defined by $(\gamma \cdot \mu)\left\{\gamma^{\prime}\right\}=\mu\left\{\gamma^{\prime} \gamma^{-1}\right\}$.
} 
Because of Proposition [1.6 it suffices to demonstrate that $\left\|g_{m}(x)-g_{m}(y)\right\| \leq$ $1 /\left(2 m^{2}\right)$ in two cases: first in the case that $d(x, y) \leq m$, and second in the case that $y=\gamma^{\prime} x$ with $\gamma^{\prime} \in \Gamma,\left|\gamma^{\prime}\right| \leq m$. In the first case we may write

$$
\left\|g_{m}(x)-g_{m}(y)\right\| \leq \sum_{\gamma} \mu_{n_{1}}\{\gamma\}\left\|f_{n_{2}}(\gamma x)-f_{n_{2}}(\gamma y)\right\| .
$$

By hypothesis we have $d(\gamma x, \gamma y) \leq R$ for each term appearing on the right and therefore each term $\left\|f_{n_{2}}(\gamma x)-f_{n_{2}}(\gamma y)\right\|$ is bounded by $(2 m)^{-2}$. Since $\sum_{\gamma} \mu_{n}\{\gamma\}=1$ the desired result follows. In the second case write, by a simple change of variable,

$$
\left\|g_{m}(x)-g_{m}\left(\gamma^{\prime} x\right)\right\| \leq \sum_{\gamma}\left|\mu_{n_{1}}\{\gamma\}-\mu_{n_{2}}\left\{\gamma\left(\gamma^{\prime}\right)^{-1}\right\}\right|\left\|f_{n_{2}}(\gamma x)\right\| .
$$

By hypothesis,

$$
\sum_{\gamma}\left|\mu_{n_{1}}\{\gamma\}-\mu_{n_{2}}\left\{\gamma\left(\gamma^{\prime}\right)^{-1}\right\}\right| \leq(2 m)^{-2}
$$

This gives the result in the second case also.

Let $\Gamma$ be a (discrete) group that acts on a compact Hausdorff space $Y$. Recall that one says that the action of $\Gamma$ is amenable if there exists a sequence of weak- $*$ continuous maps $\mu_{n}: Y \rightarrow \operatorname{Prob}(\Gamma)$ such that, for each $\gamma \in \Gamma$,

$$
\sup _{y \in Y}\left\|\gamma \cdot \mu_{n}(y)-\mu_{n}(\gamma y)\right\| \rightarrow 0
$$

as $n \rightarrow \infty$. Thus a group $\Gamma$ is amenable if and only if its action on a point is amenable. We may and shall assume that for each $n$ there is some finite subset of $\Gamma$ on which all the measures $\mu_{n}(y)$ are supported.

3.3 Remark It is clear that the definitions of property A and of amenable action are closely related. In fact, the main result of 4 is that a group $\Gamma$ has property A if and only if its natural action on its Stone-Cech compactification is amenable.

We want to generalize Corollary 3.2 to the case of amenable actions.

3.4 Theorem Suppose that $Y$ is a compact manifold, or a finite simplicial complex, and that the group $\Gamma$ acts on $Y$ amenably by Lipschitz homeomorphisms. Then the warped cone $X=\mathcal{O}_{\Gamma}(Y)$ has property $A$. 
Proof It will suffice to prove property A at infinity. The proof is an elaboration of the proof of Proposition 3.1] Let $f_{n}: X \rightarrow \operatorname{Prob}(X)$ be the functions that are provided by the definition of property A. Let $\mu_{n}$ be a Følner sequence of maps $Y \rightarrow \operatorname{Prob}(\Gamma)$, as in the definition of amenable action, and extend them radially to maps $X \rightarrow \operatorname{Prob}(\Gamma)$ (because we are only concerned with property A at infinity we do not need to worry about what happened near the cone point). As before, define functions $g$ on $X$ by 'convolving' $\mu$ with $f$ :

$$
g_{m}(x)=\sum_{\gamma \in \Gamma} \mu_{n_{1}}(x)\{\gamma\} f_{n_{2}}(\gamma x),
$$

where $n_{1}$ and $n_{2}$ are chosen by the same procedure as in the earlier proof. As before these are $d_{\Gamma}$-uniformly localized weak-* continuous maps from $X$ to $\operatorname{Prob}(X)$. To complete the proof of property A at infinity we must demonstrate the 'approximate invariance' condition that for fixed $s$,

$$
\inf _{K \subseteq X \text { compact }} \sup _{d(x, y)<s, x, y \notin K}\left\|g_{m}(x)-g_{m}(y)\right\| \rightarrow 0
$$

as $n \rightarrow \infty$.

Because of Proposition [1.6] it suffices to demonstrate the desired convergence separately in two cases: first in the case that $d(x, y) \leq s$, and second in the case that $y=\gamma^{\prime} x$ with $\gamma^{\prime} \in \Gamma,\left|\gamma^{\prime}\right| \leq s$. In the first case we may write

$$
\begin{aligned}
\left\|g_{m}(x)-g_{m}(y)\right\| \leq \sum_{\gamma} \mu_{n_{1}}(x)\{\gamma\} & \left\|f_{n_{2}}(\gamma x)-f_{n_{2}}(\gamma y)\right\| \\
& +\sum_{\gamma}\left|\mu_{n_{1}}(x)\{\gamma\}-\mu_{n_{1}}(y)\{\gamma\}\right|\left\|f_{n_{2}}(\gamma y)\right\| .
\end{aligned}
$$

The first sum on the right-hand side tends to zero by the same argument as before. As for the second sum, notice that because of the weak-* continuity of $\mu_{n_{1}}$ when considered as a map on $Y$, together with the geometry of the $d$-metric on the open cone, there is for any $\varepsilon>0$ a compact subset $K$ of $X$ such that $\left|\mu_{n_{1}}(x)\{\gamma\}-\mu_{n_{1}}(y)\{\gamma\}\right|<\varepsilon$ for $x, y \notin K, d(x, y)<s$. Thus the second sum may be made arbitrarily small by restricting $x, y$ to lie outside a compact set $K$. This completes the proof in the first case.

In the second case $y=\gamma^{\prime} x$ write, by a simple change of variable,

$$
g_{m}(x)-g_{m}\left(\gamma^{\prime} x\right)=\sum_{\gamma}\left(\mu_{n_{1}}(x)\{\gamma\}-\mu_{n_{1}}\left(\gamma^{\prime} x\right)\left\{\gamma\left(\gamma^{\prime}\right)^{-1}\right\}\right) f_{n_{2}}(\gamma x) .
$$

By definition of a $F \varnothing l n e r$ sequence the term in parentheses tends to 0 in norm, uniformly for $\left|\gamma^{\prime}\right| \leq s$. This gives the result in the second case also. 


\section{Non property A examples}

In this section we will consider examples of the following sort. Let $G$ be a compact Lie group, and let $\Gamma \leq G$ be a dense finitely generated subgroup, which we consider as a discrete group acting on $G$ by left translation. We study the coarse geometry of the warped cone $\mathcal{O}_{\Gamma}(G)$.

4.1 Proposition Suppose that the warped cone $\mathcal{O}_{\Gamma}(G)$, as defined above, has property $A$. Then $\Gamma$ is amenable.

Proof Since $X=\mathcal{O}_{\Gamma}(G)$ has property A, there is a sequence $\left\{k_{n}\right\}$ of continuous positive type kernels on $X$ satisfying the conditions of Proposition 2.3 . By averaging with respect to the Haar measure on $G$, we may assume that these kernels are invariant under left multiplication by $G$; in particular, they are invariant under the (left translation) action of $\Gamma$. Thus for each $t>0$ there is a function $h_{n, r}$ on $\Gamma$ of positive type such that

$$
k_{n}((r, g),(r, \gamma g))=h_{n, r}(\gamma)
$$

for every $g \in G$.

The functions $h_{n, r}$ are all bounded in absolute value by 1 and therefore we can find a function $h_{n}$ on $\Gamma$ of positive type such that

$$
h_{n, r_{k}}(\gamma) \rightarrow h_{n}(\gamma)
$$

for a suitable sequence $r_{k} \rightarrow \infty$.

Fix $\gamma$. For all sufficiently large $r$, the warped distance from $(r, g)$ to $(r, \gamma g)$ is precisely the word length of $\gamma$. Since $k_{n}$ has controlled support, it follows that $h_{n}(\gamma)=0$ whenever the word length of $\gamma$ is sufficiently large. In other words, $h_{n}$ has compact support. Furthermore, since $k_{n} \rightarrow 1$ uniformly on controlled sets as $n \rightarrow \infty$, we have $h_{n} \rightarrow 1$ pointwise. Thus $\Gamma$ admits a sequence of positive definite functions of compact support tending pointwise to 1 , and this is a well-known characterization of amenability.

4.2 Remark From this result and Theorem 3.4 it follows that if $\Gamma$ acts amenably on $G$, then $\Gamma$ itself is amenable. This is easy to prove directly.

4.3 Remark It is well known that $G=S O(3)$ contains a free subgroup $\Gamma$ on two generators. Thus $\mathcal{O}_{\Gamma}(G)$ is a coarse space that does not have property A.

By a similar device we may prove: 
4.4 Proposition Suppose that the warped cone $\mathcal{O}_{\Gamma}(G)$, as defined above, is uniformly embeddable in Hilbert space. Then $\Gamma$ has the Haagerup property [1]. In particular, if $\Gamma$ has property $T$, then $\mathcal{O}_{\Gamma}(G)$ cannot be uniformly embedded in Hilbert space.

Examples of dense property $\mathrm{T}$ subgroups of compact Lie groups can be found for instance with $G=S O(5)$; see [5]. In the sequel we will show that some of the resulting warped cones are counterexamples to the coarse Baum-Connes conjecture.

Proof We use the same idea as the preceding proof. To say that $X=\mathcal{O}_{\Gamma}(G)$ admits a uniform embedding into Hilbert space is to say that $X$ admits a continuous negative type kernel $k$ which is effective, that is, there are unbounded increasing functions $\rho_{1}, \rho_{2}: \mathbb{R}^{+} \rightarrow \mathbb{R}^{+}$such that

$$
\rho_{1}\left(d\left(x, x^{\prime}\right)\right) \leq k\left(x, x^{\prime}\right) \leq \rho_{2}\left(d\left(x, x^{\prime}\right)\right) .
$$

Averaging over $G$, we may assume that $k$ is $G$-invariant. For a fixed $\gamma$,

$$
h_{r}(\gamma)=k((r, x),(r, \gamma x))
$$

depends only on $\gamma$ and is bounded by $\rho_{2}(|\gamma|)$; so we can find a sequence $r_{k} \rightarrow \infty$ such that the limit

$$
h(\gamma)=\lim _{k} h_{r_{k}}(\gamma)
$$

exists. It is a negative type function and satisfies $h(\gamma) \geq \rho_{1}(|\gamma|)$, so it is proper. Thus $\Gamma$ admits a proper negative type function, which is the Haagerup property.

Compare these results with Propositions 11.26 and 11.39 in [8].

\section{References}

[1] P-A Cherix, M Cowling, P Jolissaint, P Julg, A Valette, Groups with the Haagerup property, Progress in Mathematics 197, Birkhäuser Verlag, Basel (2001) MathReview

[2] N Higson, J Roe, Warped cones and the coarse Baum-Connes conjecture (2004), in preparation

[3] N Higson, E K Pedersen, J Roe, $C^{*}$-algebras and controlled topology, $K$-Theory 11 (1997) 209-239 MathReview

[4] N Higson, J Roe, Amenable group actions and the Novikov conjecture, J. Reine Angew. Math. 519 (2000) 143-153 MathReview 
[5] A Lubotzky, Discrete Groups, Expanding Graphs and Invariant Measures, Progress in Mathematics 125, Birkhäuser, Basel-Boston-Berlin (1994) MathReview

[6] J Roe, From foliations to coarse geometry and back, from: "Analysis and geometry in foliated manifolds", (X Masa, E Macias-Virgós, JA Alvarez López, editors), World Scientific (1995) 195-206 MathReview

[7] J Roe, Index Theory, Coarse Geometry, and the Topology of Manifolds, CBMS Conference Proceedings 90, American Mathematical Society, Providence, R.I. (1996) MathReview

[8] J Roe, Lectures on Coarse Geometry, University Lecture Series 31, American Mathematical Society (2003) MathReview

[9] J-L Tu, Remarks on Yu's "property A" for discrete metric spaces and groups, Bull. Soc. Math. France 129 (2001) 115-139 MathReview

[10] G Yu, The coarse Baum-Connes conjecture for spaces which admit a uniform embedding into Hilbert space, Invent. Math. 139 (2000) 201-240 MathReview 